\title{
EXPERIMENTS ON THE PROPAGATION OF THE INDUCTION ACROSS THE BLIND SPOT (3)*
}

\author{
TAROW INDOW, TAKAAKI KOYAZU, AND TAKASHI KOZAKI
}

\author{
Keio University
}

In previous studies $(\mathbf{2}, \mathbf{3})$, it was shown that, in so far as the phenomena of induction are concerned, the blind spot became functionally equivalent to other retinal regions when a stimulus pattern (proximal) falling across the blind spot gave rise to a continuous figure in perception. That is to say, under such a condition, propagating induction not only traversed the blind spot but also was reflected, refracted and absorbed by the appropriate figures which were perceived "across the blind spot". Besides, no remarkable difference was observed in the refractive index and also in the index of absorption respectively whether these were determined in the region corresponding to the blind spot or in other regions of the retina. When no figure was perceived " across the blind spot ", however, propagation of induction was blocked by the blind spot as discovered by Motokawa (6). The present experiment was designed to investigate whether the blind spot is functionally equivalent to other regions of the retina, if it becomes a traversable medium for induction, also with respect to the velocity of the propagation. In other words, the pro-

* STUdies on the INDUCTION IN VISUAL PROCESS TAKING ELECTRICAL PHOSPHENE AS AN INDEX (6). The authors wish to express their warmest thanks to Prof. Motokawa of Tohoku University for his continuing interest throughout the series of investigations. This study was financed by the Ministry of Education. blems were: (a) to study whether the velocity can be determined with considerable accuracy even along the path running across the blind spot; (b) to determine whether the induction traverses the blind spot with the same velocity as in other regions of the retina.

The propagation velocity has been repeatedly measured with various methods in Motokawa's laboratory $(\mathbf{4}, \mathbf{5}$, 6, 7, 8, 9, 10, 11), and highly consistent results have been obtained to give the value between $1.69 \mathrm{~mm}$. and $1.77 \mathrm{~mm}$. per sec. in the dark-adapted retina assuming the distance from the posterior nodal point to the retina as $17 \mathrm{~mm}$. It was also discovered that the velocity was practically constant irrespective of regions of the retina and of directions of the propagation $(\mathbf{5}, \mathbf{7}, \mathbf{9}, \mathbf{1 1})$. Furthermore, it became clear that neither color nor intensity of the stimulus to evoke the induction affected the velocity $(\mathbf{5}, \mathbf{7}, \mathbf{9})$. As can be inferred from the fact that the beam of induction is refracted by the retinal region adapted to a prism-shaped colored luminous figure, however, the velocity depends upon the state of adaptation of the retina. Compared with the velocity in the darkadapted region, the velocity in the region adapted to white light is higher and that in the region adapted to colored light is lower. It may be said, therefore, on the analogy of the terminology in optics, that the adaptation to white light makes the retinal region less dense and the adaptation to clored light makes the retinal region denser. 
Since the refraction does occur in the region corresponding to the blind spot (3), the part of the blind spot underlying the perception of a continuous colored figure must be denser than the remaining part in the blind spot. It is not known, however, whether there is any difference in the density between the blind spot as a whole and other retinal regions, and this is one of the problems to be investigated in the present study. Taking into account the fact that approximately the same refractive index was obtained in the blind spot as well as in other retinal regions $(\mathbf{3})$, the difference, if any, in the velocity is expected to be fairly small. Hence, it seems indispensable for the present experiment to employ a highly accurate method in measuring the velocity. As stated above, one of the purposes of this study lies in testing the accuracy with which the propagating velocity of induction is measured.

\section{Experimental Procedures}

The phenomena concerning induction can be observed by measuring a change in the excitability of the eye to electrical stimulation when the eye is illumiated, prior to the delivery of the electrical stimulus, by various luminous figures, chromatic and achromatic. The electrical excitability of the eye is always given by the reciprocal of the minimum voltage of constant pulse of 0.1 sec. in duration to cause the phosphene in the peripheral field of vision of $S$. The electrical stimulus is applied to the right eye through a pair of silver electrodes; one placed on the forehead slightly above the eyebrow and the other on the homolateral temple of $S$. Let us denote by $E_{0}$ the excitability of the dark-adapted eye for at least 20 minutes. The effect of a pre-illuminating light upon the excitability is always expressed in terms of $\zeta=\left(E-E_{0}\right) / E_{0} \times 100$ where $E$ denotes the excitability determined after the eye is pre-illuminated. As the excitability is enhanced by the pre-illumination, $\zeta$ is positive. Let us denote by $\zeta_{B}$ the excitability obtained with the preillumination of a white luminous patch of $2 \mathrm{sec}$. in duration and by $\zeta_{-A}$ the excitability determined with the same white patch when it is preceded by one or more chromatic luminous figures, namely, when the eye is stimulated by one or more colored lights and by a white patch in succession prior to the delivery of the electrical pulse. Under appropriate conditions, $\zeta_{A}$ appears considerably greater than $\zeta_{B}$. The difference $\left(\zeta_{A}-\zeta_{B}\right)$ is defined as representing the induction of the preceding chromatic stimulus upon the white patch. According to Motokawa, $\left(\varsigma_{A}-\zeta_{B}\right)$ will hereafter be called "contrast effect" (CE). In Fig. 1 are shown together all the pre-illuminating figures which are to be presented in the temporal sequence as given in the inset of Fig. 1. The induction will be caused by the yellow figures $(Y)$ at the point where the white patch $(W)$ is presented. However, if the propagating induction which is to start from the blue figure $(B)$ arrives at the point before $W$ is presented, the induction will disappear as a consequence of neutralization (6). It is Motokawa's ingenious idea to detect arrival or non-arrival at the point of $\mathrm{W}$ of the induction starting from $B$ by absence or presence of the induction of $\mathrm{Y}$ respectively. For this reason, the yellow figures will be called Detector and the blue figure Inducer according to Motokawa's terminology. The white figure, Sc, is a device to produce a beam of propagating induction and also to prevent the induction from directly arriving at $\mathrm{W}$. It is well known that induction of any kind is reflected by the region once stimulated or being stimulated by a white figure. The white figure, $R$, is a reflector which the beam strikes upon. When the fixation mark $\mathrm{X}_{1}$ is fixated, a part of $R$ forms the image in the blind spot. Nevertheless, it is perceived as a whole in the shape as given in Fig. 1. The white bar, $\mathrm{Bb}$ is to block the propaga- 


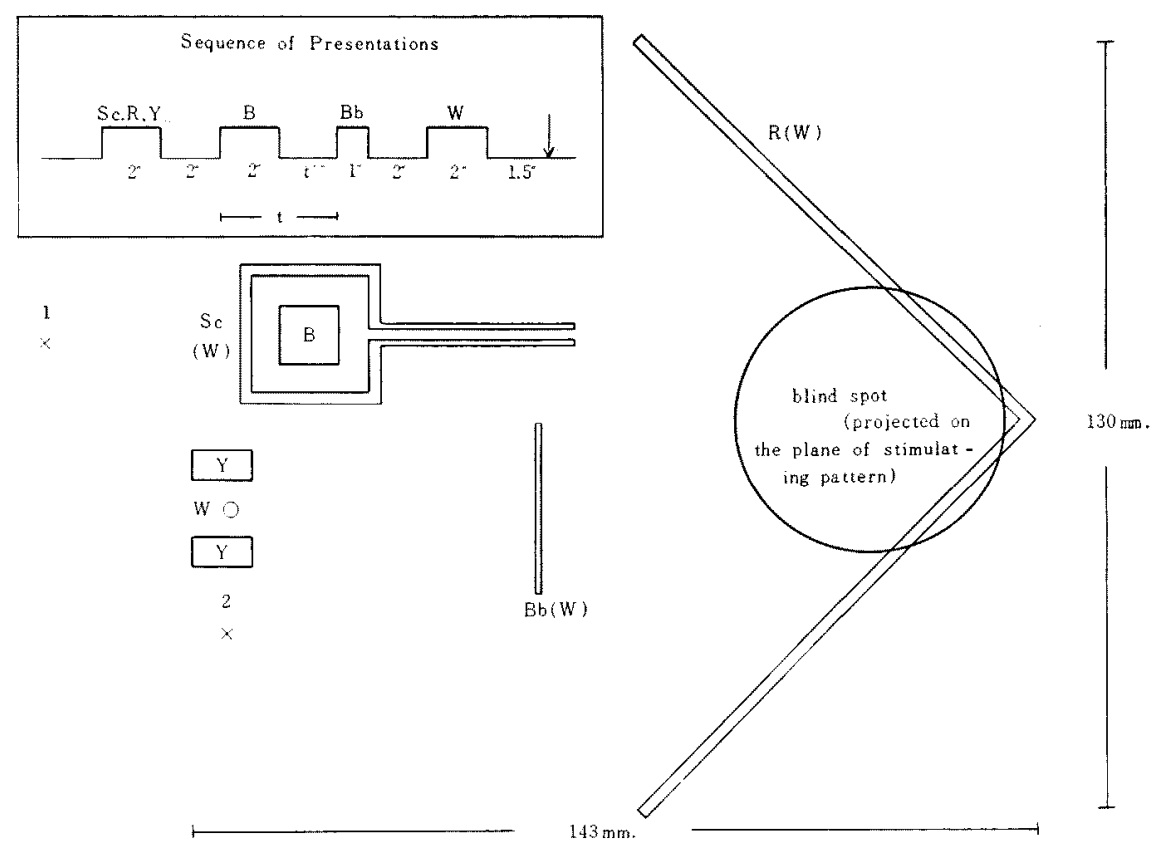

Fig. 1

tion of the induction. Its position was varied systematically along the path of propagating induction in order to test whether or not the beam had passed the position before it was presented. If the beam of the induction propagating has passed the position, neutralization will be observed at $W$, i.e., $C E=0$, whereas, if $\mathrm{Bb}$ is presented prior to the arrival of the beam, it will block the propagation and consequently the induction of $\mathrm{Y}$ will be observed at $W$, i.e., $C E>0$. The interval of time, $t$, between presentations of $\mathrm{I}$ and of $\mathrm{Bb}$ was either 3 or 3.5 or $4 \mathrm{sec}$. If, under a particular value of $t$, the critical length of the path along which the induction propagates for the interval of $t$ is experimentally determined, the velocity of induction will be calculated under the assumption that the velocity does not change along the path. Here the critical length means the length of the distance between the right side of $\mathrm{B}$ and the position of $\mathrm{Bb}$ at which the induction propagating is just blocked. This distance will be denoted hereafter by $d$.

A number of laws concerning induction and neutralization were the requisites indispensable to the design of the present experiment. For example, it has been repeatedly ascertained that the interval of time between cessation of Detector and presentation of W can be prolonged up to $20 \mathrm{sec}$. without any appreciable change in amount of $\mathrm{CE}$. Otherwise, it would have been impossible to measure the velocity of induction by the direct method as simple as described above. The laws utilized in experiments of this kind were enumerated in detail in a previous article (3). The psychophysical procedures of determining the minimum voltage of the electrical stimulus to give rise to the phosphene were also fully described in the same article. Because only presence or absence of the indirect induction of $Y$ was the question at issue, the electrical pulse was delivered at $1.5 \mathrm{sec}$. from cessation of $\mathrm{W}$ all through this study. For the sake of comparison, the velocity of induction was 
Table 1

Fixating mark 1. Blind spot involved.

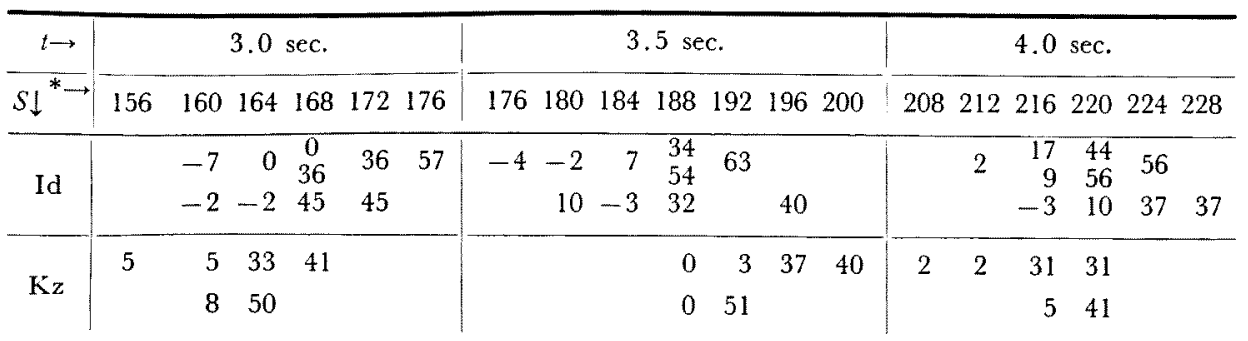

measured under the condition that the blind spot did not lie on the path of the propagation. The fixation mark, $\mathrm{X}_{2}$ in Fig. I was fixated so that the blind spot, if projected to the plane of stimulating patterns, lay on the right side of the vertex of $R$. The region of the retina through which the induction propagates necessarily differs in the two conditions; the path of the induction lies on the more peripheral region when the blind spot is involved than when not involved. Hence, if a difference is found between the velocities obtained in fixating the mark 1 and in fixating the mark 2, the difference, especially when small, will not be accounted for with unambiguity. It may suggest either that as a medium for the induction there is a difference between the blind spot and the other regions or that the velocity differs somewhat in various regions of the retina. Nevertheless, the experiment seemed to the investigators worth while carrying out, because their interest lay more in studying whether the velocity can be successfully determined even when the blind spot is involved than in making a precise comparison of the velocities in the blind spot and in other regions of the retina.

The distance was $56 \mathrm{~cm}$. from the right eye of $S$ to the transparent screen through which the rays were delivered as the preilluminating stimuli. The illumination of the screen was 106 luces and when the stimulus limen was taken as an unit, the intensities of transparent lights, i.e., $Y, B$, and $W$ were as follows respectively: $6.7 \times 10^{6}$,
$8.0 \times 10^{6}$, and $1.5 \times 10^{3}$ when the mark 1 was fixated, and $2.6 \times 10^{6}, 1.7 \times 10^{6}$, and $1.9 \times 10^{3}$ when the mark 2 was fixated. The stimulus limens were determined with the two $S$ s participating and the averages were defined as the units. Although three $S$ s were employed in the present study, the data of one $S$ was discarded for the reason stated later. The remaining two $S$ s are highly experienced in observing the phosphene.

\section{Results, Analysis, and Discussion}

The blind spot becomes a traversable medium for induction whenever perception of a continuous figure of any direction, not necessarily in the direction of the propagation, takes place " across the blind spot". It is remarkable and of a great theoretical importance that such a small portion of $\mathrm{R}$ in the blind spot as indicated in Fig. 1 was shown sufficient to make the blind spot loose its blocking effect towards the induction. As a matter of course, $R$ was perceived in its complete form and traversing the blind spot of the induction was apparently due to the continuity of $R$ " across the blind spot". Suffice it to say that, when fixation was slightly shifted to the right so as to remove the whole $\mathrm{R}$ from the blind spot, the induction was blocked by the blind spot lying on its path.

The whole data, i.e., CE's determined for obtaining the critical distance, $d$, were given in Tables 1 and 2 . As 
Table 2

Fixating mark 2. Blind spot not involved.

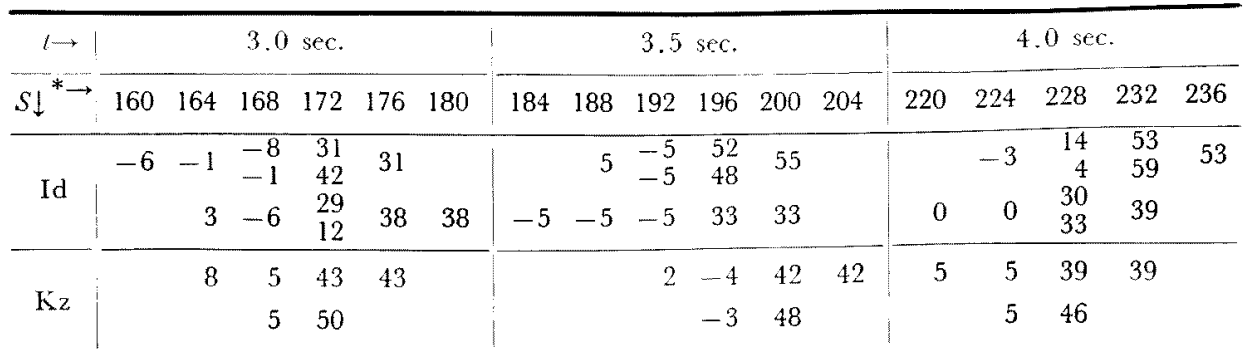

* Distance between the right side of $B$ and $B_{b}$ in $m m$.

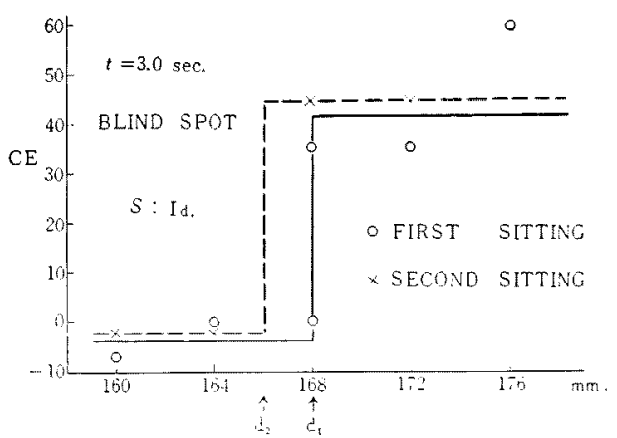

Fig. 2

stated above, when the mark 1 was fixated, the blind spot lay on the path of the propagating induction, and when the mark 2 was fixated, the blind spot was not involved. As an example, from Table 1 CE's in the case of "blind spot involved", Id, and $t=3 \mathrm{sec}$. are plotted in Fig. 2. The bar, Bb, was presented in this example at four different positions to make four distances in the abscissa. Measurements were repeated in two different experimental sittings in all the cases. In the first sitting the position of $\mathrm{Bb}$ was systematically varied in $4 \mathrm{~mm}$. step for probing the critical point at which an abrupt change appeared in the magnitude of CE. Following this procedure was unavoidable in the first sitting because $\mathrm{E}$ had no notion about the position where the discontinuity occurs. In the case of
Id, once the position of the abrupt change had been determined, around that point $\mathrm{Bb}$ was presented in the second sitting at four or five positions, $4 \mathrm{~mm}$. apart from one to the next, in a randomized order. The discontinuity in CE was still obtained in this procedure though a shift of the critical point was observed sometimes. When occurred, the shift never exceeded $4 \mathrm{~mm}$., however. It is to be noted that the induction was always observed at distances larger than the critical length of path and the neutralization always appeared at distances shorter than that. The induction and neutralization never intermingled even in the measurements carried out in a randomized order. The magnitude of CE fluctuated considerably, especially in different sittings, which did not obscure however the discontinuity in the magnitude of $\mathrm{CE}$. Ordinarily, the critical distance $d$ was estimated at the middle of the distances; the smallest distance where the induction was obtained and the largest one where the neutralization occurred. Measurements were sometimes repeated in vicinities of the critical point. When the case happened that either the induction and also the neutralization occurred at the same distance or the magnitude of $\mathrm{CE}$ intermediate between the case of induction and that of ncu- 


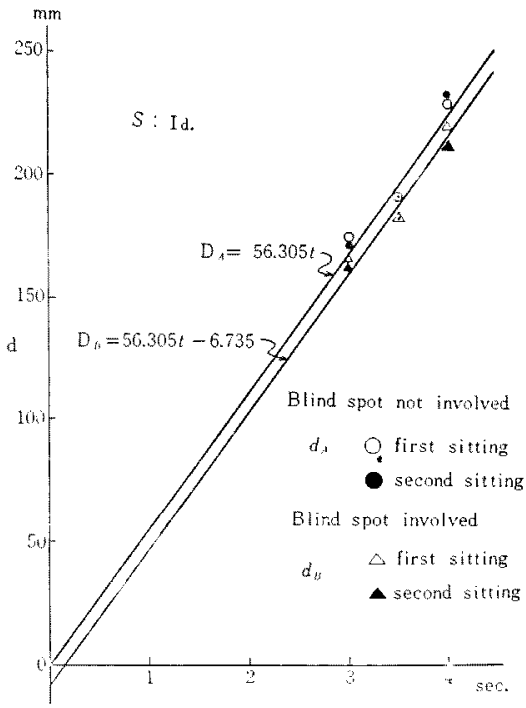

Fig. 3

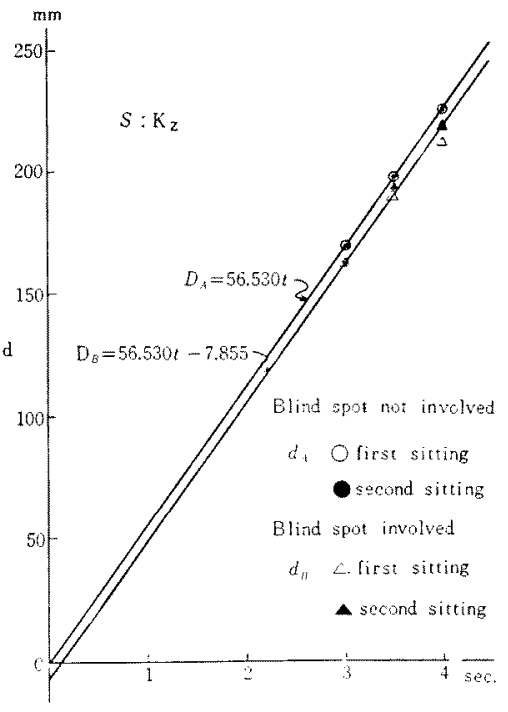

Fig. 4 tralization appeared, then $d$ was defined at that distance. In the case of $\mathrm{Kz}$, measurements were repeated in the second sitting only in vicinities of the critical distance. Three $S_{\mathrm{s}}$ were run on this experiment, but the results of one $S$ did not establish with certainty the determination of the critical point, and the report was made of the remaining two $S \mathrm{~s}$ ' data in the article. One of the investigators participated only as an experimenter.

The critical distance $d$ thus determined is plotted in Fig. 3 and Fig. 4 against the interval of time $t$ during which the induction is supposed to have propagated. Let us denote by $d_{-A}$ the critical distance under the condition that the blind spot is not involved and by $d_{B}$ the one under the condition that the blind spot is involved. The former was indicated by a circle and the latter by a triangle in the figures. Though slight, $d_{. A}$ 's are systematically larger than the corresponding $d_{B}$ 's so that a common line can not be fitted to both. With respect to $d_{A}$, it will be natural to assume a straight line passing through the origin. That is equivalent to assume that the latency for the induction to start from B and also the latency for the induction propagated to wipe out the induction of $\mathrm{Y}$ existing at $\mathrm{W}$ are negligible compared with the time necessary for the propagation. This is the fact repeatedly ascertained in Motokawa's laboratory $(\mathbf{5}, \mathbf{7}, \mathbf{1 1})$. What line should be fitted to $d_{B}$ then? In this case, the medium through which the induction propagates is divided into two kinds; the blind spot (B-part) and the other regions of the retina ( $\mathrm{N}$-part). Then, with respect to the propagating velocity, $B$-part and $N$-part may differ to each other though these are homogeneous within each respectively. Should it hold, as the induction propagates in order through $N_{1}, B$, and $N_{2}$, the relationship between $t$ and $d_{B}$ will be as given in Fig. 5 where the velocity is assumed smaller in B than in N. What was obtainable in this experiment was only the relationship in $\mathrm{N}_{2}$, i. e., the segment drawn in a bold stroke. Hence, 
the line to be fitted to $d_{B}$ 's must be straight and of the same slope as the straight line for $d_{A}$ 's but its intercept with the ordinate may not be zero. The intercept is negative if the velocity is smaller in B than in N. Thus, a set of the equations

$$
\begin{aligned}
& D_{A}=a t \\
& D_{B}=a t+b
\end{aligned}
$$

was fitted to $d_{A}$ 's and $d_{B}$ 's respectively as shown in Fig. 3 and 4 . Because of the peculiar form of the equations (1), modifications had to be made to the ordinary procedures of the fitting and

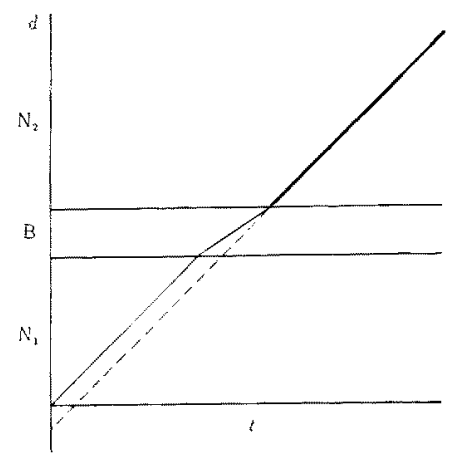

Fig. 5

also of the subsequent analysis. It might be well, therefore, to describe the procedures in general terms with a brief outline of the derivations.

Let us consider a set of equations :

$$
\begin{aligned}
& y_{1 i j}=\mu_{1 i}+\varepsilon_{1 i j} \\
& y_{2 i j}=\mu_{2 i}+\varepsilon_{2 i j}
\end{aligned}
$$

where $i=1,2, \ldots, n, j=1,2, \ldots, m$, and $\varepsilon$ is a random error which is assumed distributed $\mathcal{N}\left(0, \sigma^{2}\right)$. In accordance with (1),

$$
\begin{aligned}
& \mu_{1 i}=\alpha x_{i} \\
& \mu_{2 i}=\alpha x_{i}+\beta
\end{aligned}
$$

and the fitted straight lines are written as follows:

$$
\begin{aligned}
& Y_{1}=a x \\
& Y_{2}=a x+b
\end{aligned}
$$

Then, $a$ and $b$ will be given by the prin- ciple of least squares in the following way.

$$
\begin{aligned}
& Q=\sum_{i} \sum_{j}\left\{\left(y_{1 i j}-a x_{i}\right)^{2}\right. \\
& \left.+\left(y_{2 i j}-a x_{i}-b\right)^{2}\right\} \\
& \quad \frac{\partial Q}{\partial a}=-2 \sum_{i} \sum_{j}\left\{\left(y_{1 i j}-a x_{i}\right) x_{i}\right. \\
& \left.+\left(y_{2 i j}-a x_{i}-b\right) x_{i}\right\} \\
& \therefore \quad \sum_{i}\left\{\left(\bar{y}_{1 i}-a x_{i}\right) x_{i}\right. \\
& \left.\quad+\left(\bar{y}_{2 i}-a x_{i}-b\right) x_{i}\right\}=0
\end{aligned}
$$

where

$$
\bar{y}_{1 i}=\frac{1}{m} \sum_{j} y_{1 i j} \quad \bar{y}_{2 i}=\frac{1}{m} \sum_{j} y_{2 i j}
$$

and

$$
\begin{aligned}
& \frac{\partial Q}{\partial b}=-2 \sum_{i} \sum_{j}\left(y_{2 i j}-a x_{i}-b\right) \\
\therefore \quad & \sum_{i}\left(\bar{y}_{2 i}-a x_{i}-b\right)=0
\end{aligned}
$$

Equations (6) and (7) reduce to

$$
b=\frac{\left.\sum_{i} x_{i}-a \bar{y}_{1 i}+\bar{y}_{2 i}\right)-n \bar{x} \bar{y}_{2}}{S_{x}}
$$

where

$$
\begin{aligned}
\bar{y}_{2} & =\frac{1}{m n} \sum_{i} \sum_{j} y_{2 i j} \quad \bar{x}=\frac{1}{n} \sum_{i} x_{i} \\
S_{x} & =2 \sum_{i} x_{i}^{2}-n \bar{x}^{2}
\end{aligned}
$$

Thus, the values of $a$ and $b$ given in Fig. 3 and 4 were determined by means of (8) and (9). It is to be noted that the following equations hold from (6) and (7) :

$$
\begin{gathered}
\sum_{i}\left(\bar{y}_{2 i}-Y_{2 i}\right)=0 \\
\sum_{i}\left\{\left(\bar{y}_{1 i}-Y_{1 i}\right) x_{i}+\left(\bar{y}_{2 i}-Y_{2 i}\right) x_{i}\right\}=0
\end{gathered}
$$

Now the procedures used in the analysis of covariance will be described. Let us denote by $S$ the sum of squares of the error terms. Obviously its degree of freedom, $\phi$, is $2 \mathrm{~nm}$ and it is easily shown that $S$ is divided into two independent components :

$$
\begin{gathered}
S=\sum_{i} \sum_{j}\left\{\left(y_{1 i j}-\mu_{1 i}\right)^{2}\right. \\
\left.+\left(y_{2 i j}-\mu_{2}\right)^{2}\right\}=S_{\tilde{y}}+S_{e}
\end{gathered}
$$




$$
\begin{gathered}
S_{\bar{y}}=m \sum_{i}\left\{\left(\bar{y}_{1 i}-\mu_{1 i}\right)^{2}\right. \\
\left.+\left(\bar{y}_{2 i}-\mu_{2 i}\right)^{2}\right\} \\
\phi=2 n \\
S_{e}=\sum_{i} \sum_{j}\left\{\left(y_{1 i j}-\bar{y}_{1 i}\right)^{2}\right. \\
\left.+\left(y_{\Omega i j}-\bar{y}_{2 i}\right)^{2}\right\} \\
\phi=2 n(m-1)
\end{gathered}
$$

An estimate for the error variance $\sigma^{2}$ is thus given by

$$
u^{2}=\frac{S_{e}}{2 n(m-1)}
$$

On the other hand, $S_{y}$ can be further analyzed :

$$
\begin{aligned}
& S_{\bar{y}}=m \sum_{i}\left[\left\{\left(\bar{y}_{1 i}-Y_{1 i}\right)+\left(Y_{1 i}-\mu_{1 i}\right)\right\}^{2}\right. \\
& \left.+\left\{\left(\bar{y}_{2 i}-Y_{2 i}\right)+\left(Y_{2 i}-\mu_{2 i}\right)\right\}^{2}\right] \\
& =m \sum_{i}\left[\left\{\left(\bar{y}_{1 i}-Y_{1 i}\right)+(a-\alpha) x_{i}\right\}^{2}\right. \\
& \left.+\left\{\left(\bar{y}_{2 i}-Y_{2 i}\right)+(a-\alpha) x_{i}+(b-\beta)\right\}^{2}\right] \\
& =m \sum_{i}\left[\left\{\left(\bar{y}_{1 i}-Y_{1 i}\right)+(a-\alpha) x_{i}\right\}^{2}\right. \\
& +\left\{\left(\bar{y}_{2 i}-Y_{2 i}\right)+(a-\alpha)\left(x_{i}-\bar{x}\right)\right. \\
& \left.+(a-\alpha) \bar{x}+(b-\beta)\}^{2}\right] \\
& =m \sum_{i}\left[\left\{\left(\bar{y}_{1 i}-Y_{1 i}\right)+(a-\alpha) x_{i}\right\}^{2}\right. \\
& +\left\{\left(\bar{y}_{2 i}-Y_{2 i}\right)+(a-\alpha)\left(x_{i}-\bar{x}\right)\right. \\
& \left.\left.+\left(\bar{Y}_{2}-\bar{\mu}_{2}\right)\right\}^{2}\right] \\
& =m \sum_{i}\left\{\left(\bar{y}_{1 i}-Y_{1 i}\right)^{2}+\left(\bar{y}_{2 i}-Y_{2 i}\right)^{2}\right\} \\
& +(a-\alpha)^{2} m \sum_{i}\left\{x_{i}^{2}+\left(x_{i}-\bar{x}\right)^{2}\right\} \\
& +m n\left(\bar{Y}_{2}-\bar{\mu}_{2}\right)^{2} \\
& +2 m\left(\bar{Y}_{2}-\bar{H}_{2}\right)(a-\alpha) \sum_{i}\left(x_{i}-\bar{x}\right) \\
& +2 m(a-\alpha) \sum_{i}\left\{\left(\bar{y}_{1 i}-Y_{1 i}\right) x_{i}\right. \\
& \left.+\left(\vec{y}_{2 i}-Y_{2 i}\right) x_{i}\right\} \\
& -2 m(a-\alpha) \bar{x} \sum_{i}\left(\bar{y}_{3 i}-Y_{2 i}\right)
\end{aligned}
$$

Evidently the fourth term vanishes and so do the last two terms because of (10) and $(11)$. Then, $\mathrm{S}_{\bar{y}}$ reduces to

$$
S_{\bar{y}}=S_{l}+(a-\alpha)^{2} m S_{x}+m n\left(\bar{Y}_{2}-\bar{\mu}_{2}\right)^{2}
$$

where

$$
\begin{gathered}
S_{l}=m \sum_{i}\left\{\left(\bar{y}_{1 i}-Y_{1 i}\right)^{2}+\left(\bar{y}_{2 i}-Y_{2 i}\right)^{2}\right\} \\
\phi=2 n-2=2(n-1)
\end{gathered}
$$

and $S_{x}$ is defined before. The degree of freedom of $S_{l}$ is reduced by 2 on account of (10) and (11), and it is clear that the degree of freedom of each of the remaining two components is 1 . That is to say, the three terms of the right side of (15) are orthogonal from one another according to Cochran's theorem and each has $\sigma^{2} \chi^{2}$ distribution of its own degree of freedom. Hence,

$$
\begin{aligned}
& E\left\{\frac{S_{l}}{2(n-1)}=\sigma^{2}\right. \\
& E\left\{(a-\alpha) m S_{x}\right\}=\sigma^{2}
\end{aligned}
$$

In other words, $a$ is distributed

$$
\mathcal{N}\left\{\alpha, \frac{\sigma^{2}}{m S_{x}}\right\}
$$

Now the followings are obvious. The hypothesis regarding the form of the regression of $y$ upon $x, H_{l}$, is tested by means of

$$
\begin{aligned}
\mathrm{F}_{l} & =\frac{S_{l}}{2(n-1) u^{2}} \\
\phi & =2(n-1), 2 n(m-1)
\end{aligned}
$$

and the hypothesis that $\alpha$ is equal to a specified value $\alpha_{0}, H_{\alpha}$, is tested by

$$
\begin{gathered}
F_{a}=\frac{\left(a-\alpha_{0}\right) m S_{x}}{u^{2}} \\
\phi=1,2 n(m-1)
\end{gathered}
$$

The remaining step will be to ascertain whether $b$ is significantly different from a specified value $\beta_{0}$. For that purpose, let us consider a pair $\left\{x_{0} Y_{20}\right\}$, and using (2), (8) and (19),

$$
\begin{gathered}
Y_{20}=a x_{0}+b=a x_{0}+\bar{y}_{2}-a \bar{x}=\bar{y}_{2} \\
+a\left(x_{0}-\bar{x}\right)
\end{gathered}
$$

Hence, the variance of $Y_{20}$ is given by the equation $(\mathbf{1 1}$, p. 34):

$$
\sigma^{2}\left(\dot{x}_{20}\right)=\sigma^{2}\left(\bar{y}_{2}\right)+\left(x_{0}-\bar{x}\right)^{2} \sigma^{\prime \prime}(a)
$$

By means of (19) and remembering that $\bar{y}_{2}$ is a mean of sample of size $m n$,

$$
\begin{aligned}
\sigma^{2}\left(Y_{\mathbf{2}}\right) & =\frac{\sigma^{2}}{m n}+\frac{\left(x_{0}-\bar{x}\right)^{2} \sigma^{2}}{m S_{x}} \\
& =\frac{\sigma^{2}}{m}\left(\frac{1}{n}+\frac{\left(x_{0}-\bar{x}\right)^{2}}{S_{x}}\right)
\end{aligned}
$$

When $x_{0}=0, \quad Y_{20}=b$, the hypothesis under discussion, $H_{;}$, is tested by 
Table 3

\begin{tabular}{l|l|l|l}
\hline & & \multicolumn{1}{|c}{$\mathrm{Id}$} & \multicolumn{1}{|c|}{$\mathrm{Kz}$} \\
\hline $\mathrm{H}_{l} ;$ linearity of regression & $\phi=4,6$ & $\mathrm{~F}_{l}=12.68^{* *}$ & $\mathrm{~F}_{l}=0.90$ \\
$\mathrm{H}_{\alpha} ; \alpha=56.00 \mathrm{~mm}$. & $\phi=1,6$ & $\mathrm{~F}_{\alpha}=9.87$ & $\mathrm{~F}_{\alpha}=15.01 * *$ \\
$\mathrm{H}_{\beta} ; \beta=0$ & $\phi=1,6$ & $\mathrm{~F}_{\beta}=59.18^{* *}$ & $\mathrm{~F}_{\beta}=40.25^{* *}$
\end{tabular}

** The hypothesis is rejected with the level of significance of 0.01 .

$$
\begin{gathered}
F_{\dot{\beta}}=-m^{2} \\
u^{2}\left(\frac{1}{n}+\frac{\bar{x}^{2}}{S_{x}}\right) \\
\phi=1,2 n(m-1) .
\end{gathered}
$$

The estimate for the error variance $\sigma^{2}$ was $2.33 \mathrm{~mm}$. for Id and $2.66 \mathrm{~mm}$. for $\mathrm{Kz}$. The results of testing the statistical hypotheses are summarized in Table 3. In the case of Id, the variation of $d$ 's about the regression line happened to be significant statistically. None the less, the standard deviation of the variation was as small as $5.45 \mathrm{~mm}$., being of the size of the interval by which the position of $\mathrm{Bb}$ was varied. Referring to the propagation velocity determined by Motokawa's, when $t=1.0$ sec. and in the plane $56 \mathrm{~cm}$. apart from the eye, the critical distance was expected to be $56.00 \mathrm{~cm}$. Although the difference for $\mathrm{Kz},\left(\alpha-\alpha_{0}\right)=0.53 \mathrm{~mm}$, was statistically significant, it is obvious that the velocity obtained in this $S$ is also fairly in line with that obtained in Motokawa's experiments. Concerning with $\mathrm{H}_{\beta}$, doubtlessly the intercept $\beta$ is of negative.

The difference observed between the two lines, $D_{A}$ and $D_{B}$, calls for some speculation. One reasonable hypothesis might be that the fact $\beta<0$ indicates, as shown in Fig. 5 and stated before, the smaller velocity in the blind spot (B-part) than in other regions of the retina (N-part). But, this hypothesis should not be stressed too much because the difference between the velocities is so small that it may be accounted for by local variation, if any, of the propagation velocity along the path. As to the density for induction, the retina or its corresponding cortical region may not be a completely homogeneous medium. Though slight, a systematic change was observed by Saito among the velocities determined in various regions of the retina $(\mathbf{1 1})$. It is to be noted, however, the velocity was greater in the periphery than in the central region. In the present experiment, the path lies in more peripheral part of the retina when the blind spot is involved $\left(D_{B}\right)$ than when not involved $\left(D_{A}\right)$. Hence, the result $\beta<0$ is not easily explained from the local variation of the velocity found in Saito's experiment. In personal communication, Motokawa suggested an interesting hypothesis. By traversing the blind spot, in his opinion, the induction of $B$ seems to be "weakened" in the sense that the latency for the induction becomes longer to give rise to the neutralization at W. Obviously, further studies are required before a satisfactory $\mathrm{ex}$ planation is offered to the difference discovered between the induction traversing the blind spot and the induction propagating in other regions of the retina.

\section{SUMMARY}

1. The propagating velocity of the so-called induction was measured along the two paths, the one running across 
the blind spot and the other lying in other regions of the retina.

2. Again was confirmed the previous observation that the blind spot changes to a traversable medium for induction when a proximal stimulus falling across the blind spot gives rise to a continuous figure in perception.

3. The velocity was determined with considerable accuracy along both paths with two $S$ s. Projected into the plane $56 \mathrm{~cm}$. apart from the right eye, the velocities were $56.3 \mathrm{~mm}$. and 56.5 $\mathrm{mm}$. per sec. for the two $S$ s respectively when the blind spot was not involved. These coincided closely with the values so far obtained in Motokawa's laboratory.

4. A fact was observed that seems to suggest some kind of difference between the blind spot and other regions of the retina as mediums for the induction to propagate.

\section{References}

1. Acron, F. S. Analysis of straight-line data. John Wiley, 1959.

2. Indow, T., Kuno, U., \& Yoshida, T. Studies on the induction in risual process taking the electrical phosphene as an index (2).-Experiments on the propagation of the induction across the blind spot (1). Psychologia, 1958, 1, 175-181.

3. Indow, T., Koyazv, T., \& Yoshid, T. Studies on the induction in visual process taking electrical phosphene as an index
15).-Experiments on the propagation of the induction across the blind spot (2). Jap. psychol. Res., 1959, No. 7, 17-28.

4. Latayama, S., \& Aizawa, K. The mechanism of spatial induction in the retina. Tohoku F. exp. Med., 1956, 64, 179-187.

5. Motokawa, $\mathbf{K}$. Wave nature of retinal processes and field of vision. NisshinIgaku, 1951, 38, (1), 131-141. (2), 211222. (In Japanese).

6. Motokswa, K. Propagation of retinal induction. J. Neurophysiol., 1951, 14, 339-351.

7. Motokawa, K., Iwama, K., \& Ebe, M. Velocities of spreading induction in human and mammalian retinas. Tohoku 7. exp. Med., 1954, 59, 11-22.

8. Motokawa, K., \& Komatsu, M. Reflection of spreading induction in the human retina. Tohoku 7. exp. Med., $1957,67,29-41$.

9. Motokawa, K., \& Komatsu, M. Propagation velocity and total reflection of spreading induction in the light-adapted human retina. Tohoku 7. exp. Med., $1958,67,149-158$.

10. Motokawa, K., Ebe, M., \& Komatsu, M. Reflection of spreading induction in the retina. Tohoku f. exp. Med., 1958, 68, $139-149$

11. Sarto, T. Conduction velocities of spreading induction in various regions of the human retina. Tohoku 7 . exp. Med., 1958, 69, 13-23.

(Receired December, 20, 1959) 